\title{
Structure integrated shape memory polymer composites for multi- dimensional forming
}

\author{
Tristan Timmel $^{1, a^{*}}$, Alexander Hackert ${ }^{2, b}$, Tomasz Osiecki ${ }^{2, c}$, Colin Gerstenberger ${ }^{2, d}$, Lothar Kroll ${ }^{2, e}$ \\ ${ }^{1}$ Cluster of Excellence MERGE, Technische Universität Chemnitz, 09107 Chemnitz, Germany \\ ${ }^{2}$ Institute of Lightweight Structures, Technische Universität Chemnitz, 09107 Chemnitz, Germany
}

\begin{abstract}
Large-scale curved structures such as wind turbine wings usually require a special and cost intensive transport to the installation destination. These transport and installation costs can be reduced by a flat transport condition and the possibility of layering several structural components. For this reason, the focus at the Department of Lightweight Structures and Polymer Technology at TU Chemnitz was on a novel active material composite, which enables resource-efficient mass production and has a new component architecture. The large-volume multidimensional curvature of the active structure could be achieved by using a shape memory polymer (SMP). The associated reduction of the specific investment costs, the use of materials and the possibility of an integrative design, can contribute to the fact that, for example, the small wind turbines will become an economically viable investment in the future. The active structure influencing was represented by means of a finite element simulation (FEM) for different material composites and could be verified by generic demonstrators regarding its validity.
\end{abstract}

\section{Introduction}

Today most commonly used technological production of material compounds is the composite construction which combine self-sufficient materials, each with specific properties, in a construction using the right material in the right place. The materials are connected by a joining process using filler material. The mixed construction is thus characterized by a joining zone between the composite material systems of a composite material. This procedure can be realized especially by the differential construction. Due to the advantageous combination of different materials, the overall properties of an additively connected assembly should be improved. In this case, a material can dominate in its specific properties. The primary goal of using the mixed structure is to reduce the mass of an assembly while increasing performance. This increase is characterized by increasing the rigidity, the strength and the toughness and by improving the vibration resistance [1]. In addition, mixed construction solutions can be used to meet special customer requirements. In order to realize a cost-effective and mass-production-compatible structural element, an in-line production with continuous production is desirable. The goal is the development of an active composite material that can form a multi-dimensional structure with the help of an active matrix component. The multidimensional shaping is triggered by an external excitation, whereby the matrix component reacts with a change in length.

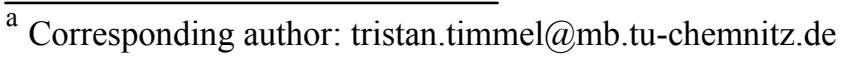

\section{Active material compound}

A particularly suitable matrix material is the shape memory polymer Desmopan ${ }^{\circledR}$ DP 2795A-SMP of the Bayer AG subsidiary Covestro Deutschland AG, which serve the plastics sector. This active component of the developed material composite controls the shaping behavior of the structure. The passive component is in the first step, a Polypropylene (PP) film which is replaced in the second step by a textile component, see Figure 1.

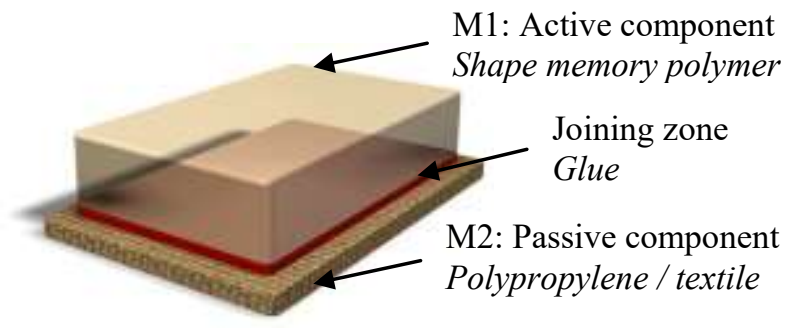

Figure 1. Illustration of the active material compound.

The textile material used for all variants is a pliable, impregnated plain weave cotton fabric, in which the warp and weft threads are made of twisted yarns. This textile fabric made of natural fiber with a surface weight of $320 \mathrm{~g} / \mathrm{m}^{2}$, as well as linen or hemp cloths, is used as sail cloth in the boat industry. The selected cotton fabric has a high tear strength, good weather resistance, resistance to UV radiation and good dimensional stability and ductility. 
In addition, the textile is mildew resistant, dirt and water repellent. The decision to use the cotton fabric is based on the fact that this textile with the required properties is cheap and available in large quantities on the market. Figure 1 shows the two selected composite components, which are bonded by means of the joining process adhesive bonding to form the active material compound. This has the advantage that possibly occurring shear stresses in the interface can be reduced or compensated by the use of a suitable adhesive. The risk of delamination of the composite material can thus be minimized and the composite properties are controlled.

\section{Structure formation}

\subsection{Analytical consideration}

The basis for the modeling of the generic technology demonstrators is the mathematical description of the active material composite. According to the functional diagram shown in Figure 2, the composite can be transformed into a model of a contracting actuator and a linear compression spring.
a) Block diagram
b) Schematic illustration
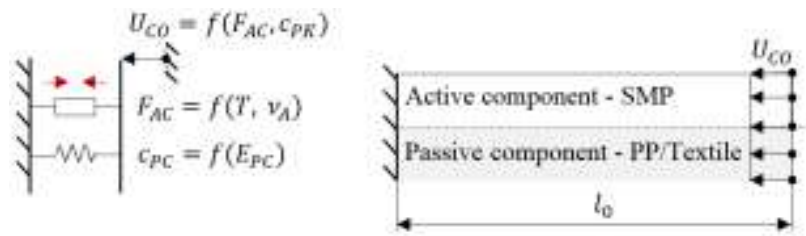

Figure 2. Functional diagram of active material combination.

Starting from the equilibrium condition that the contraction force of the active component $F_{A K}$ of the force of the passive component $F_{P K}$, the contraction shift of the active composite $U_{C O}$ can be described below

$U_{C O}=F_{P C} / c_{P C}=\left(l_{0} \cdot A_{A C} \cdot\left(K_{\sigma l} \cdot T-K_{\sigma 2}\right)\right) /\left(E_{P C} \cdot A_{P C}\right)$.

The desired strain (contraction) of the active composite $S_{C O}$ is given in the following:

$S_{C O}=U_{C O} / l_{0}=\left(A_{A C} \cdot\left(K_{\sigma 1} \cdot T-K_{\sigma 2}\right)\right) /\left(E_{P C} \cdot A_{P C}\right)$.

The resulting deflection due to the induced structure curvature is subsequently derived analytically. The analytical solution is based on the thermal expansion problem in bimetallic beams, see Figure 3. The radius of the active component $R_{A C}$ is proportional to the radius of the second component $R_{P C}$ and equal in the region of the joint zone. The two radii are summarized approximately to a radius of curvature $\mathrm{R}_{\mathrm{C}}$

$$
R_{A C} \sim R_{P C}=R_{C}
$$

With the approach of Eq. (3), the same curvatures $\kappa$ are subsequently produced in the actuator layer and the second component.

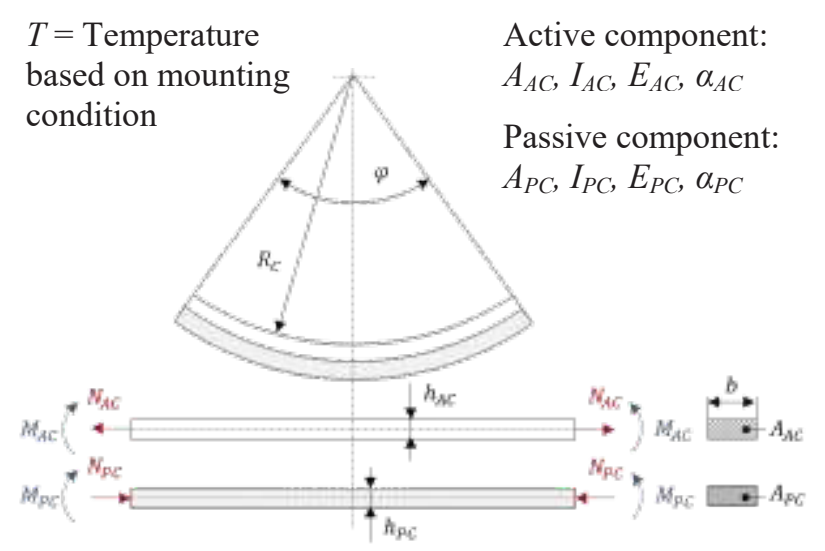

Figure 3. Thermal bending model.

The product of the modulus of elasticity $E$ of the respective component and the area moment of inertia $I$ gives the bending stiffness, which in both layers is defined as the ratio of bending moment to curvature $M / \kappa$

$\kappa=1 / R_{C}=M_{A C} /\left(I_{A C} \cdot E_{A C}\right)=M_{P C} /\left(I_{P C} \cdot E_{P C}\right)$

The bond is defined by a longitudinal force equilibrium at the structural ends with the longitudinal force of the active component $N_{A C}$ and the longitudinal force of the passive component $N_{P C}$. The sum of the bending moment of the active component $M_{A C}$ and the passive component $M_{P K}$ gives the total bending moment of the composite $M$

$N_{A C}=N_{P C}=N \rightarrow N \cdot\left(h_{A C} \cdot h_{P C}\right) / 2=M_{A C}+M_{P C}=M(5)$

The displacement compatibility at the interface with the thermal expansion coefficients $\alpha$ of the respective component as well as the cross-sectional areas $A_{A C}$ and $A_{P C}$

$$
\begin{aligned}
& \alpha_{A C} \cdot \Delta T+N /\left(E_{A C} \cdot A_{A C}\right)+h_{A C} / 2 \cdot 1 / R_{C} \\
& \alpha_{P C} \cdot \Delta T-N /\left(E_{P C} \cdot A_{P C}\right)-h_{P C} / 2 \cdot 1 / R_{C}
\end{aligned}
$$

can be formulated with the moment of inertia of the active component $I_{A C}$ for the desired structure curvature.

After replacing the thermal expansion difference by the contraction of the active component from Eq. (2) taking into account the thermal expansion of the second component, the resulting actuation strain $\Lambda$ is given in Eq. (8)

$$
\begin{gathered}
\Lambda=\alpha_{P C} \cdot \Delta T+S_{C O} f(T) \\
\Lambda=\alpha_{P C} \cdot\left(T-R_{T}\right)-\left(A_{A C} \cdot\left(K_{\sigma l} \cdot T-K_{\sigma 2}\right)\right) /\left(E_{P C} \cdot A_{P C}\right)
\end{gathered}
$$

and the sought-after active structural behavior in Eq. (9)

$$
\begin{aligned}
& 1 / R_{C}=\left(\alpha_{P C} \cdot\left(T-R_{T}\right)-A_{A C} /\left(E_{P C} \cdot A_{P C}\right) \cdot\left(K_{\sigma 1} \cdot T-K_{\sigma 2}\right)\right) \\
& /\left[2 /\left(h_{A C}+h_{P C}\right) \cdot I_{A C} / A_{A C} \cdot\left(1+\left(I_{P C} \cdot E_{P C}\right) /\left(I_{A C} \cdot E_{A C}\right)\right)\right. \\
& \left.\cdot\left(1+\left(A_{A C} \cdot E_{A C}\right) /\left(A_{P C} \cdot E_{P C}\right)\right)+\left(h_{A C}+h_{P C}\right) / 2\right] .
\end{aligned}
$$


The deflection $U_{\text {bend }}$ of the active cantilever is shown schematically in Figure 4 and can be determined with the help of Eq. (10).

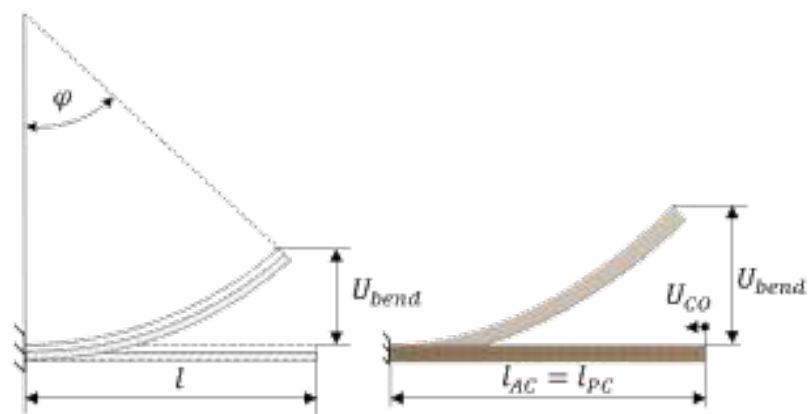

Figure 4. Bending model.

The deflection $U_{b e n d}$ is defined using the angle of curvature $\varphi$ below:

$$
\begin{gathered}
U_{\text {bend }}=d \varphi / d x \cdot l^{\wedge} 2 / 2 \\
d \varphi / d x=\text { const. }=1 / R_{C} \rightarrow 1 / R_{C} \cdot l^{\wedge} 2 / 2 .
\end{gathered}
$$

In summary, it can be stated that the structural properties of the second component have a major influence on the structure curvature of the composite material due to a change in length of the active component.

\subsection{Numerical consideration}

The material composite demonstrator, which is to be used for the simulation of structural shaping, consists of two components, which are connected to each other by means of an adhesive. The active component consists of the SMP Desmopan DP 2795A-SMP and has the task of producing a deflection of the material composite in the $\mathrm{Z}$ direction by a controlled change in length.

The second component represents the passive cover layer of the composite material and consists of a textile fabric. The geometry and dimensions of the composite material demonstrator result from the SMP tensile specimens according to DIN EN ISO 27, type 1A. The desired structure curvature of the composite material, as a result of a change in length of the active component, requires contacting of the two components in at least two points. With increasing contact, up to the full-surface connection, an increase in the degree of deformation and a more uniform curvature is expected.

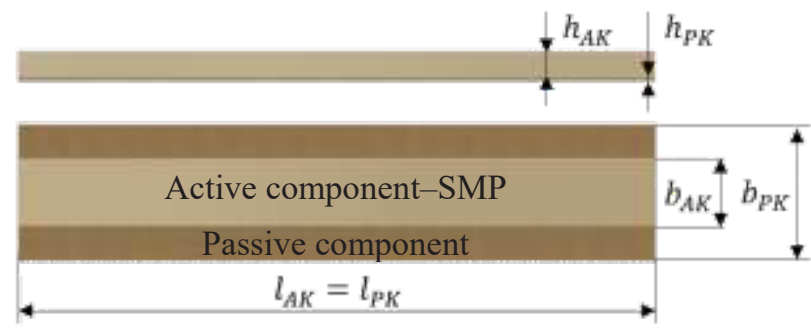

Figure 5. Dimensions of the generic technology demonstrator
The active component of the generic composite material demonstrator is first heated to a temperature above the switching temperature $T_{g}$ in order to solve the physical crosslinking (secondary valence bonds). In this thermally activated state, the SMP is elastically deformable [2]. The Desmopan DP 2795A-SMP tensile bars are heated in the climate chamber of the Zwick / Roell Z250 static double-axis tensile testing machine from Zwick / Roell and stretched by $2.85 \mathrm{~mm}$ in a straincontrolled manner, see Step 2 in Figure 6. By means of the multiXtens tactile extensometer, exact and reproducible stretching is possible. By cooling the SMP below the switching temperature $T_{g}$, the temporary shape of the tensile specimen can be frozen by the formation of physical crosslinks and the concomitant increase in rigidity in the hard segment of the block copolymer [3]. Subsequently, the tensile specimen is cut to the specified length dimension $l_{A C}$ and joined to form a composite material.

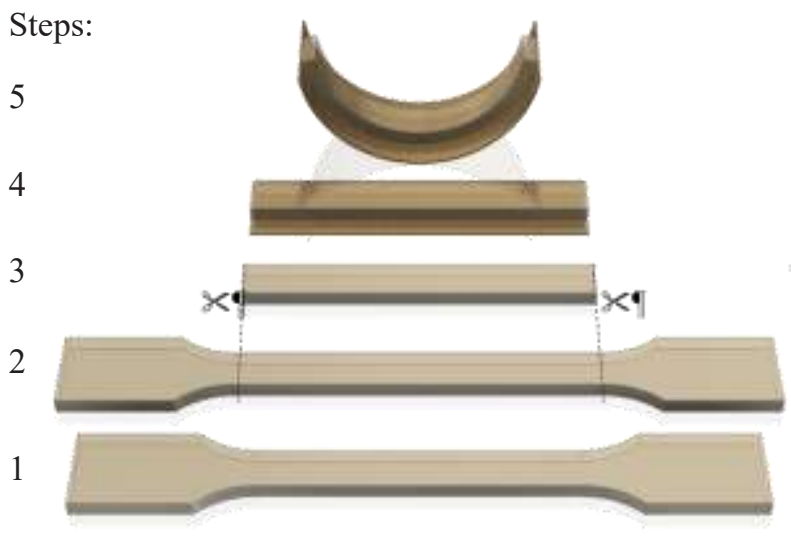

Figure 6. Creation of the generic technology demonstrator

Subsequently, when the SMP is reheated to a temperature greater than $T_{g}$, the physical crosslinks dissolve again, returning the tensile sample to its permanent shape. Step 5 in Figure 6 symbolically shows the resulting deflection due to the contraction of the SMP by $2.85 \mathrm{~mm}$ for a composite material variant, which is evaluated in the next step. In the following composite material variant in Figure 7, the SMP component is bonded to the entire surface of the $0.5 \mathrm{~mm}$ thick polypropylene film (PP). Due to the predetermined temperature change of $30 \mathrm{~K}$, the simulated contraction of the SMP is $2.85 \mathrm{~mm}$ and leads to a deflection in the $\mathrm{Z}$ direction of $41 \mathrm{~mm}$.

Numerical results: $\mathrm{z}$-displacement in [mm]

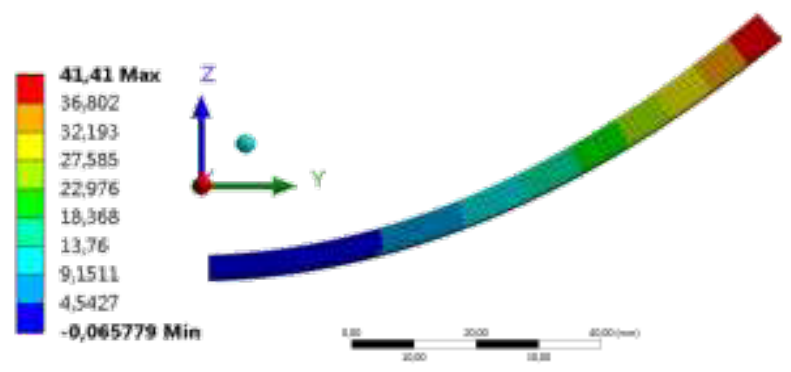

Figure 7. SMP-glue-PP - Full-surface contact. 
The analytical consideration has already shown that when using a nearly limp component an induced structure curvature of the composite material due to a change in length of the active component is theoretically not possible. Despite the use of a nearly limp component, a structural formation on the generic demonstrators can be watch. This is due to the adhesive used. The glue impregnates the fibers of the textile semifinished product in the region of the joining zone, which means that the fibers are protected against buckling under a compressive load (compression). This leads locally to an increase in the E-module in this composite material. The adhesive thus assumes the function of the matrix in a composite material or a fiber-reinforced plastic composite. A further increase in the modulus of elasticity is accompanied by the increase in the strength of the adhesive due to the chemical reaction in the curing process. The resulting Zdisplacement of the textile material composite with a fullsurface adhesive layer between the two components is shown in Figure 8.

Numerical results: z-displacement in [mm]

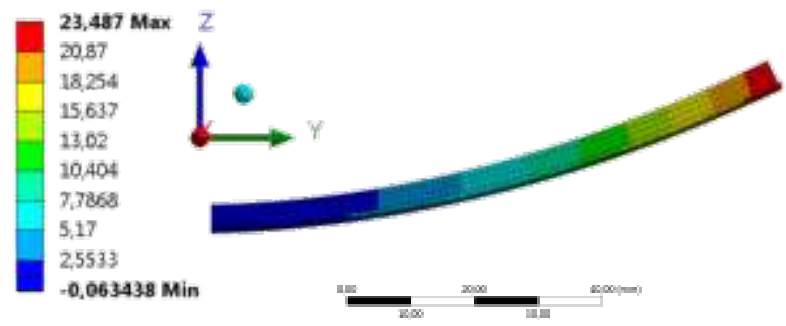

Figure 8. SMP-glue-textile - Full-surface contact.

\subsection{Experimental consideration}

The evaluation of the experimentally determined Zdisplacement for the generic composite material with PP film and textile fabric as cover layer is shown in Figure 9 and also in Figure 10 as a box plot. In the experimental studies the generic demonstrators with full-surface contact showed a large structural formation in the $\mathrm{Z}$ direction.

Experimental results: z-displacement in [mm]

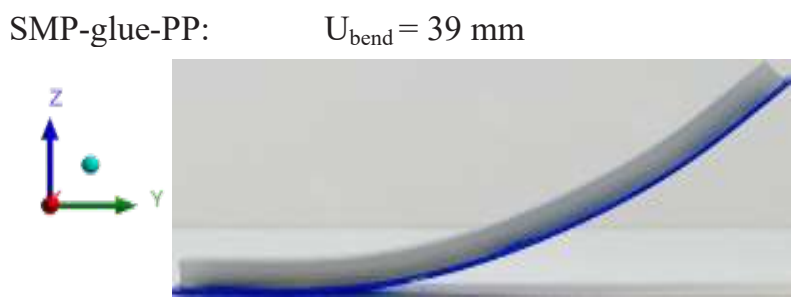

SMP-glue-textile: $\quad \mathrm{U}_{\text {bend }}=25 \mathrm{~mm}$

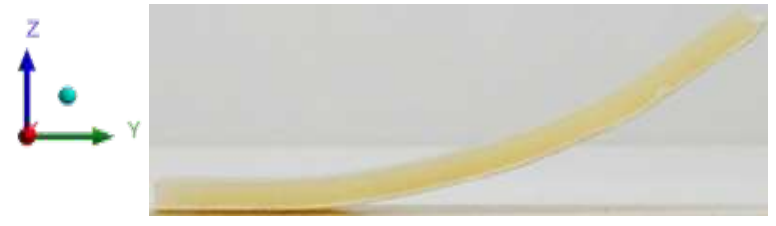

Figure 9. SMP-Glue-Textile - Full-surface contact.
These two composite material variants are compared with respect to the Z-displacement.

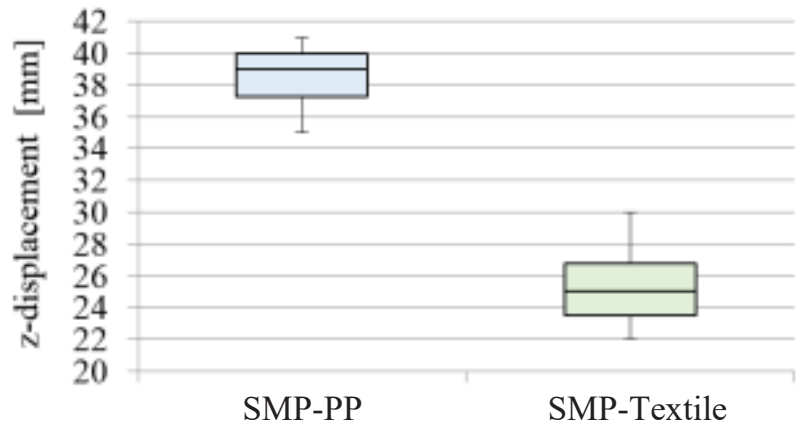

Figure 10. Evaluation of the collected z-displacement.

The deviation of the Z-displacement in the range of $22 \mathrm{~mm}$ to $30 \mathrm{~mm}$ is due to the different degree of impregnation of the textile with adhesive and the associated layer thickness of the adhesive. The Z-shift of the textile material composite is on average 35\% lower than that of the isotropic material composite with PP film. The reason for this lies in the textile properties and can be adjusted by, for example, the type of fabric, thread and stitch density. The validity of the finite element model could also be proven for the textile application. For initial estimates of the structural behavior of active material composites, the FEM model used is sufficiently accurate and thus forms the basis for future investigations.

\section{Summary}

The use of a textile wind turbine blade with active structural curvature shown in Figure 11 is one way that can be used in the near future. The advantages that speak for the use of a textile wind turbine blade, in addition to the low weight and ease of manufacture, the possibility of functional integration and personalization. The functional integration can be implemented easily and inexpensively by means of embroidery technology and adhesive technology. The back injection technology is also a possibility to integrate sensors or actuators with a biobased thermoplastic material [4] on the textile component.

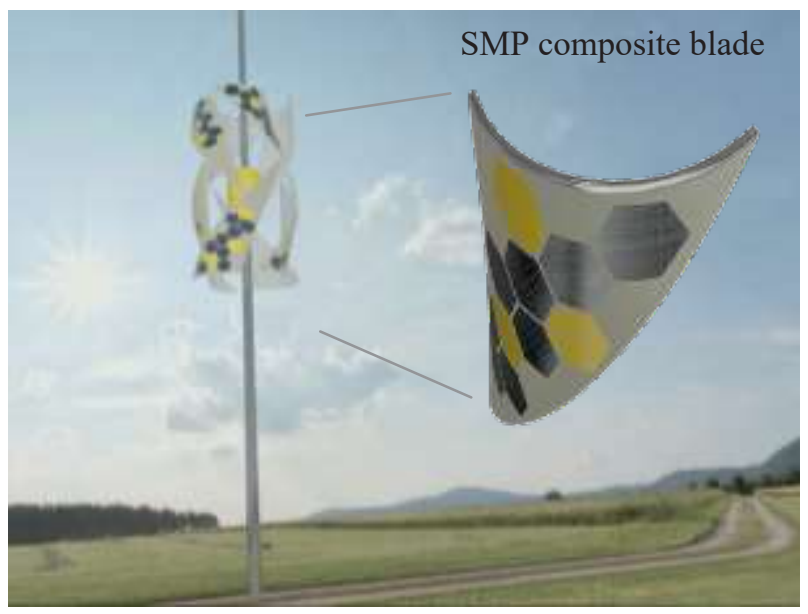

Figure 11. Small wind turbine with SMP composite blades. 
Sensors such as embroidered strain gauges, temperature sensors or moisture-detecting sensors can be applied by means of embroidery on the textile. The SWT can thus simultaneously function as a weather station. Design features and personalization options such as actively illuminating fibers or Q-LED lighting elements are currently the subject of research at the Chemnitz University of Technology and can also be applied to the textile sail for future applications. The use of flexible photovoltaic cells is also conceivable on the textile wind turbine wing. Due to the vertical clamping of the sails, the diameter of the rotor is reduced, whereby the doubleclamped sword segments are subjected to a bending and thus cause a buckling of the individual sails [5]. The applied SMP profiles on the canvas support the stretchedout shape and help to prevent the sail breaking through in headwinds. In addition to the sail shape stability, the profiles have the task to guide the incident wind at an optimum angle to the sail area. By horizontally directed laminar flow and forwarding this in the wind-averted wing, the efficiency of the rotor can be significantly improved. The fundamental advantage of the developed textile shape memory polymer wind turbine blade is the

\section{References}

1. T. Timmel, A. Hackert, T. Osiecki, C. Gerstenberger, L. Kroll, Combined Injection Molding Technology for Dynamically Stressed Multi-Material Coupling Elements, Key Engineering Materials, vol. 744, pp. 322-326, (2017)

2. M. Behl, A. Lendlein, Shape Memory Polymers, Materials Today 10, vol. 4, pp. 20-28, (2007)

3. C, Schmidt, Werkstoffwissenschaftliche Untersuchungen zur Verarbeitung von FormgedächtnisPolymeren und zur funktionellen Ermüdung bei zyklischer Abfrage des Einwegeffekts, PhD, (2011) even transport state, which allows stratification of many wind turbine blades and thereby et al reduced transport and installation costs. The large-volume curvature of the wing thus takes place only in the assembled state at the site. The envisaged integration of the wind turbine in an already electrified and developed light mast reduces $u$. a. the costs for the foundation, the development and commissioning of the small wind turbine. In the next steps of this research project the goal is to pursue the strategy of bivalent resource efficiency (BRE strategy) more closely in order to exploit all available resource efficient.

\section{ACKNOWLEDGEMENTS}

The presented studies were performed as part of a preinvestigation within the Federal Cluster of Excellence EXC 1075 "MERGE Technologies for Multifunctional Lightweight Structures" and supported by the German Research Foundation (DFG) to explore integrative inMold processes with plastic/ metal composites. Financial support is gratefully acknowledged.

4. R. Rinberg, T. Hartmann, A. Nikiforov, L. Volfson, L. Kroll, Investigation of bio-based polyamide with short fibers for lightweight structures, Conference proceedings International MERGE Technologies Conference - IMTC 2017 Lightweight Structures, vol. 3, pp. 33-34, (2017)

5. L. Kroll, M. Heinrich, S. Müller, J. Kaufmann, FEAnalyse anisotroper Koppeleffekte mit der ESAComp-Schnittstelle zur belastungsgerechten Auslegung von Snowboards, ANSYS Conference \& 25. CADFEM Users'Meeting, vol. 25, (2007) 